\title{
Haemodialysis-associated hepatitis
}

\author{
RONALD FinN \\ M.D., M.R.C.P. \\ Liverpool Regional Urological Centre, \\ Sefton General Hospital, Liverpool 15
}

\section{Summary}

Outbreaks of hepatitis have occurred in renal units all over Europe and the U.S.A. and have carried a high mortality.

This is an iatrogenic disease, transmitted by blood from carriers of SH virus, uraemic patients often suffering subclinical attacks.

The risks to the staff of these units are so high that they should not be asked to undertake this work if outbreaks cannot be controlled.

OUTBREAKs of hepatitis have occurred in many haemodialysis and transplantation units in this country. Similar outbreaks have occurred in Europe and the United States. In many cases these outbreaks have been large and there has been a significant mortality, especially among the staff. There can therefore be no doubt that haemodialysis-associated hepatitis is a real clinical entity. As it has occurred following the introduction of haemodialysis it can be classified as an iatrogenic disease, and it is certainly the most important iatrogenic disease that we have yet produced.

\section{The Liverpool outbreak}

The Liverpool outbreak has involved fifty-seven cases. It started in 1966 and is now under control, but not extinguished, in 1970. These outbreaks tend to be large and long-lasting. Patients, staff and relatives who help in dialysis are affected, but it is typical of these outbreaks that the disease is more severe in the contacts than in the patients. There has been a variable morbidity but no mortality. We have been fortunate as other outbreaks have involved a significant mortality. This long-lasting and severe outbreak has led to a severe retardation of our haemodialysis and transplantation programmes.

\section{High density SH carriers}

One may ask why these epidemics occur, and the reason is to be found in the way SH virus is handled by normal and uraemic subjects. Most of these outbreaks are caused by the SH virus. If a normal person is infected with the SH virus, the incubation period of up to 3 months is followed by a short viraemia followed by a clinical attack of hepatitis, and following this SH antigen can no longer be detected. In contrast, the uraemic patient is unable to mount a normal clinical hepatitis and often has a subclinical attack, or even no demonstrable attack, but then proceeds to become a chronic carrier of the virus. Uraemic patients have deficient immunological responses and are unable to destroy the virus and so become carriers. These patients carry very large numbers of virus particles and may be called high density SH carriers, and this makes their blood highly infective.

\section{High blood exposure technique}

These highly dangerous SH carriers are then treated by haemodialysis which might be called a high-blood-exposure technique. Dialysis involves repeated access to the arterial and venous systems and an extracorporeal circulation. This means that despite every precaution some blood spillage is inevitable with repeated haemodialysis. It is, then, easy to see how a blood aerosol of highly infected blood settles on some exposed area and gains access to the circulation by means of some microscopic abrasion. Haemodialysis-associated hepatitis is therefore due to the combination of a high density carrier state with a high-blood-exposure technique. Once you have a uraemic SH carrier on a dialysis unit, spread is almost inevitable to dialysis staff, both medical, surgical and technical. Five members of the laboratory staff, who have never entered the unit, were affected, thus proving beyond reasonable doubt that the infection is blood-borne. If the patients are put on home dialysis spread to relatives is common. Other patients on the unit also become infected, and they may become SH carriers. We have one patient who has been an SH carrier for at least 4 years and hence these patients present a continuing hazard to staff. Even if they are dealt with by home dialysis they require occasional hospital admissions, and this constitutes a real and continuing hazard.

\section{Ethical considerations}

The risks to staff include the immediate hepatitis morbidity and mortality. Long-term theoretical 
risks include cirrhosis and hepatoma. There is always a theoretical risk that the virus will mutate and then spread into the community, thus leading to a widespread public health hazard. Contact with an SH carrier on a dialysis or transplantation unit, therefore, involves a considerable risk to the staff as the infectivity rate is high. One may, therefore, ask whether it is justifiable to ask nursing and technical staff to run this risk, and I suspect that it is not. I have eventually come to take the extreme view that we must learn to prevent these outbreaks, otherwise it will become unethical to expose healthy young staff to these risks, and haemodialysis and transplantation facilities will have to be seriously restricted. If this position be accepted, then the future of haemodialysis and renal transplantation will depend on our ability to control these outbreaks.

\section{Prevention of further outbreaks}

Fortunately I think we now have the knowledge to prevent these outbreaks. The key advance has been the clinical application of the SH antigen test as most of the serious outbreaks have been due to serum hepatitis (MS-2) (Turner \& Bruce White, 1969). Outbreaks of infective hepatitis (MS-1) occur, but they do not appear to be as serious and tend to be of short duration. All patients must be SH screened before admission to dialysis and transplantation units. The SH virus usually enters the dialysis unit by means of blood transfusion, and the amount of blood transfused must be seriously curtailed. Blood transfusion cannot be avoided completely, but when essential the blood should be screened for SH antigen. The other safeguard is only to use blood from donors who have given at least five previous donations without trouble. Because of the long incubation period infection has usually spread to other patients before it is noted that a member of staff is infected. It has, therefore, usually been found that there are several SH carriers on infected units, and this makes the task of controlling the epidemic much more difficult. The large hospital-based dialysis units are therefore highly dangerous and they should be replaced by smaller four-bedded units concentrating on home dialysis training and the holding of small transplant pools. This is an important step, and it is to be hoped that the Department of Health will revise its policy concerning the ideal size of a dialysis unit. If these measures are strictly observed, I think that future outbreaks can be largely prevented, and it need hardly be stressed that prevention is the only logical policy to adopt.

\section{Theoretical considerations}

There are several interesting theoretical aspects of these outbreaks. It is likely that what we call clinical hepatitis is an immunological battle taking place in the liver to destroy the hepatitis virus. When a patient dies of hepatitis, it is probably due to an uncontrolled immunological response, or what may be loosely termed a hypersensitivity reaction, It is, therefore, logical to treat these patients with large doses of steroids, and we have done so with good results. This brings up the whole question of treating severe inflammatory disease with steroids in addition to providing antibiotic cover. If the immunological battle is not successful the patient becomes a carrier, but comes to no harm, and this again indicates that clinical hepatitis is due to the body's reaction to the virus and that the virus is non-toxic. Uraemic patients develop tolerance to the virus and there are several other links between the liver and tolerancee Antigens presented to the spleen induce antibod formation, but antigens presented to the liver ar less likely to do so and successful liver transplantation can be carried out in the pig without immunosuppression. Somewhere in this area may be the clue to the solution of the transplant problem. Haemodialysis-associated hepatitis is undoubtedly an iatrogenic disease, and this leads one to the perhaps paradoxical realisation that the results of medical research will not always be beneficial. Powerful therapeutic techniques are inevitably accompanied by the risk of serious side-effects and iatrogenic disease is likely to become a growing problem as scientific medicine advances.

\section{Reference}

Turner, E.C. \& Bruce White, G.B. (1969) SH Antigen in haemodialysis-associated hepatitis. Lancet, ii, 121. 